\title{
p-modes of a polytropic convection zone with an overlying hot envelope
}

\author{
M. Wagner and F. Schmitz \\ Institut für Theoretische Physik und Astrophysik der Universität Würzburg, Am Hubland, 97074 Würzburg, Germany \\ e-mail: schmitz@astro.uni-wuerzburg.de
}

Received 5 April 2007 / Accepted 8 July 2007

\begin{abstract}
Aims. To investigate the behavior of non-radial stellar p-modes with high horizontal wave numbers $l$, a plane layer approximation is sufficient. In the $k-\omega$ diagram, the ridges of the p-modes are strongly influenced by the structure of the atmospheric layers. We present a one-layer model the wave equation of which can be solved in closed form. The layer consists of a polytropic convection zone smoothly joined by an envelope with exponentially increasing temperature. We investigate the behavior of p-modes. As the model is convectively unstable there are no g-modes. This shortcoming is not significant as we discuss only p-modes.

Methods. The adiabatic wave equation is reduced to Whittaker's equation. As the dispersion relation of the p-modes is a fourth order algebraic equation in $\omega$, the $\omega(k)$-relation can be given in closed form.

Results. We discuss the form of the ridges of the diagnostic diagram. It is shown that the modes concentrate at the position of the temperature minimum in the high-frequency limit. A comparision of the ridges with the ridges of a convection zone with an isothermal atmosphere is performed. In the diagnostic diagram, below the f-mode, there is a continuous spectrum. The correspondig waves behave as gravity waves in the range of the exponential temperature increase. It is discussed whether there are resonances in the continuos spectrum. Solutions of the wave equation of vertically propagating waves are presented.
\end{abstract}

Key words. hydrodynamics - waves - stars: atmospheres - stars: oscillations - sun: oscillations

\section{Introduction}

To study the behavior of stellar p-modes with high wave numbers $l$ it is sufficient to consider only the outer layers of the star. Non-radial oscillations with $l \gg 1$ extend only to the upper convection zone and to the atmospheric layers. For $l \gg 1$, the approach of these regions by a plane layer with constant gravity is common. As regards the sun, Antia \& Basu (1999) have calculated adiabatic p-modes with a real solar model. Steffens (1998) and Steffens \& Schmitz (2000) have used the plane-layer approximation. They explained the complicated behavior of the ridges in the diagnostic diagram of the solar atmosphere by variations of the atmospheric stratification. For the interpretation and discussion of the behavior of oscillations of real convection zones with real atmospheres, ideal models with closed solutions of the wave equations are helpful. Also ideal models are important to study effects as reflections of waves, cavities, resonances, pseudo-modes, the generation of waves and the excitation of modes. Thereby, the assumption of adiabaticity is common.

There are only a few one-layer models. As regards the characteristics of the propagation of adiabatic waves in an atmosphere, the isothermal atmosphere is the simplest tool. As regards the behavior of adiabatic modes of a convection zone, the simplest model is the polytropic layer already studied by Lamb (1932). For a parabolic temperature profile, Chiuderi \& Giovanardi (1979) have shown that vertical wave propagation can be described in closed form. In this case the adiabatic wave equation can be reduced to the hypergeometrical equation.

A two-layer model of the atmosphere of the earth consisting of a polytropic lower layer (troposphere) and an upper isothermal layer (stratosphere) was used by Pekeris (1948) to study the 3-dimensional propagation of waves and pulses. The standard model for the investigation of the linear dynamics of a convection zone with an atmosphere is a two-layer model consisting of a polytropic lower layer and an isothermal atmosphere. This model was often used to explain effects of wave propagation in the outer layers of the sun. References are given by Schmitz \& Steffens (1999). Above all, Price (1996) has presented a very extended study. In each layer there is a simple analytical solution of the wave equation. However, the fitting conditions do not enable a closed representation of the dispersion relation. Price has pointed emphatically to this severe shortcoming.

By use of the column mass as the independent variable, Schmitz \& Steffens (1999) finally could present a one-layer model the adiabatic wave equation of which could be solved in closed form with an analytically solvable dispersion relation. This model consits of a polytropic layer with arbitray $n$ which is smoothly joined by an asymptotically isothermal atmosphere. For the explanation of the meaning of the dubious "modes with complex frequencies" Schmitz \& Steffens (2000) this model was used successfully.

Numerous trials by the authors to find one-layer models with a wave equation solvable in closed form resulted in only one model. There, in the interior, the layer becomes polytropic with index $n=0$, i.e. a linear temperature decrease joined by an exponential temperature increase with the geometrical height. Thus, the model has a temperature minimum, but no chromospere. It represents a convection zone with a thin atmosphere and a transition layer to an arbitrarily hot corona or an envelope. Because of $n=0$ in the lower layer, the model is convectively unstable. 
As we are interested in the behavior of p-modes, the omission of the gravity waves and the occurence of unstable modes instead, plays no role. The adiabatic wave equation of our model can be reduced to a confluent hypergeometrical differential equation.

The dispersion relation of the p-modes is a forth order algebraic equation in $\omega^{2}$, thus analytically solvable. It is not the closed solution of the wave equation but above all the closed form of the $\omega(k)$ relation which justifies the presentation of such a model. The structure of the layer depends only on two parameters so that a fit to real atmospheres is restricted. The fact that the wave equation can be reduced to Whittaker's differential equation is important as this equation also describes oscillations of a purely polytropic layer. So, from a mathematical point of view, the cases are comparable. As regards the presentation of closed solutions of the wave equation and above all a dispersion relation which can be solved analytically, we think that we have reached a limit. (The one-dimensional case with only vertical propagation of waves is different.)

We do not intend to compare our results with observational data. We present the results in order to understand the influence of a modified atmosphere on the oscillations. Besides, the VAL-model is controversal. Dynamical calculations by Carlson \& Stein (1995) have shown that the chromospheric temperature rise is artificial. From our experience with the propagation of shock waves in atmospheres (Fleck \& Schmitz 1993), we share the same opinion.

In Sect. 2 we present and discuss the structure of the equilibrium layer. Section 3 deals with the reduction of the wave equation to Whittaker's differential equation. The general solution of this equation is considered in Sect. 4. The dispersion relation of the modes, a fourth order algebraic equation is derived in Sect. 5. In Sect. 6 we discuss the behavior of the dispersion curves and the trapping of the modes in the high frequency limit. Section 7 deals with relations between the modes of the present model and those of a model with an isothermal atmosphere. In Sect. 8 we discuss the continuous part of the $k-\omega$ diagram and investigate the existence of resonances. Section 9 deals with vertically propagating waves.

\section{The equilibrium layer}

Let $z$ be the vertical, outwards directed geometrical coordinate, $g$ the constant gravity, $m$ the column mass, defined by $\mathrm{d} m=-\rho \mathrm{d} z$, $p$ the pressure, $\rho$ the density, $a$ the isothermal sound speed. The equation of state is $p=a^{2} \rho$. The equilibrium condition reads $p=m g$. We put

$a^{2}(m)=\epsilon \frac{m^{2}}{m-m_{0}} \quad$ with $\quad m>m_{0}$.

The density is

$\rho(m)=\frac{g}{\epsilon} \frac{m-m_{0}}{m}$.

For $m \rightarrow \infty$ the density is constant, the layer becomes polytropic with index $n=0$. The geometrical height $z$ is

$z(m)=-\frac{\epsilon}{g}\left\{m+m_{0} \ln \left(\frac{m-m_{0}}{m_{0}}\right)\right\}+z_{0}$.

We have $m \rightarrow m_{0}$ as $z \rightarrow \infty$ and $m \rightarrow \infty$ as $z \rightarrow-\infty$. Further,

$a^{2}(z)=-g z$ as $z \rightarrow-\infty$

and

$a^{2}(z)=\epsilon m_{0} \exp \left(g z / \epsilon m_{0}\right)$ as $z \rightarrow+\infty$,

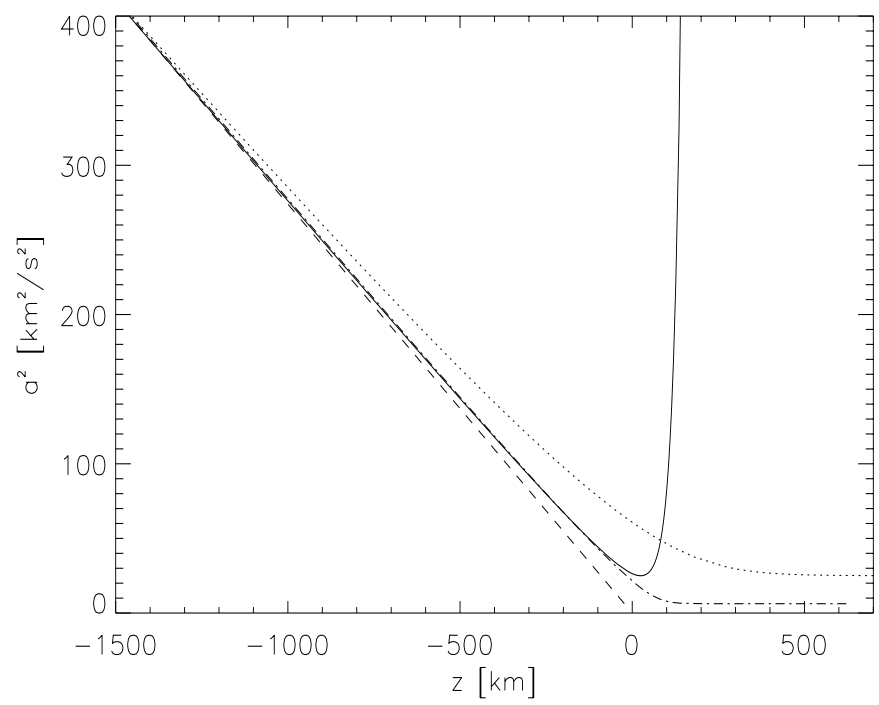

Fig. 1. Squares of isothermal sound speeds as functions of the geometrical height $z$. That of our model is represented by the solid curve, that of the pure convection zone by the dashed line. Models with isothermal atmospheres are displayed by dashed-dotted and dotted curves.

so that the sound speed increases exponentially with the geometrical height $z$. As the layer becomes polytropic with index $n=0$ for $z \rightarrow-\infty$, convective instabilities occur. The position of the minimum of the sound speed is at $m=2 m_{0}$, the onset of the convection, according to the Schwarzschild condition, is at $m=(\gamma+1) m_{0}$, where $\gamma$ is the adiabatic exponent. The fact that the configuration is convectively unstable plays no role, as we are interested in the behavior of p-modes. Also in the case of the compressible homogeneous stellar model (Pekeris 1938) convectively unstable modes occur. As regards the non-radial pmodes, however, this model is representative. It is the only stellar model, the eigenfrequencies and eigenfunctions of non-radial pulsations are given in closed form without Cowling's approximation.

We note that the parameter $\epsilon$ can be eliminated by introducing new variables $\epsilon m, \epsilon z$ and new parameters $\epsilon m_{0}, g / \epsilon$. We, however, shall not make use of this possibility.

For the illustrations, the parameters $\epsilon$ and $m_{0}$ are fitted to the temperature minimum of the VAL-C-atmosphere (Vernazza et al. 1981). With $g=2.74 \times 10^{4} \mathrm{~g} \mathrm{~cm} / \mathrm{s}^{2}$, we obtain $\epsilon=2.5 \times 10^{12}$ and $m_{0}=0.025$ in cgs-units. Figure 1 shows the square of the isothermal sound speed as a function of the geometrical height. The model has no chromosphere. The steep increase of the sound speed with $z$ may be interpreted as a transition layer. For $z \rightarrow$ $+\infty$ we have $p=m_{0} g$, i.e. a finite pressure at infinity. This is quite natural in the case of an exponential temperature increase.

For a pressure stratification $p(m)=g\left(m-m_{0}\right)$, the square of the sound speed would increase linearly with the geometrical height:

$a^{2}(z)=g z \quad$ as $\quad z \rightarrow+\infty$.

In this case the stratification becomes asymptotically polytropic with $n=0$. The wave equation cannot be solved in closed form.

The stratification (1) is a special case of the stratification

$a^{2}(m)=\epsilon \frac{m^{2}}{\left(m-m_{0}\right)^{\alpha}} \quad$ with $\quad m>m_{0}$

$z(m)=-\frac{\epsilon}{g} \frac{1}{(1-\alpha)(2-\alpha)}\left(m-m_{0}\right)^{1-\alpha}\left(m(1-\alpha)+m_{0}\right)$. 


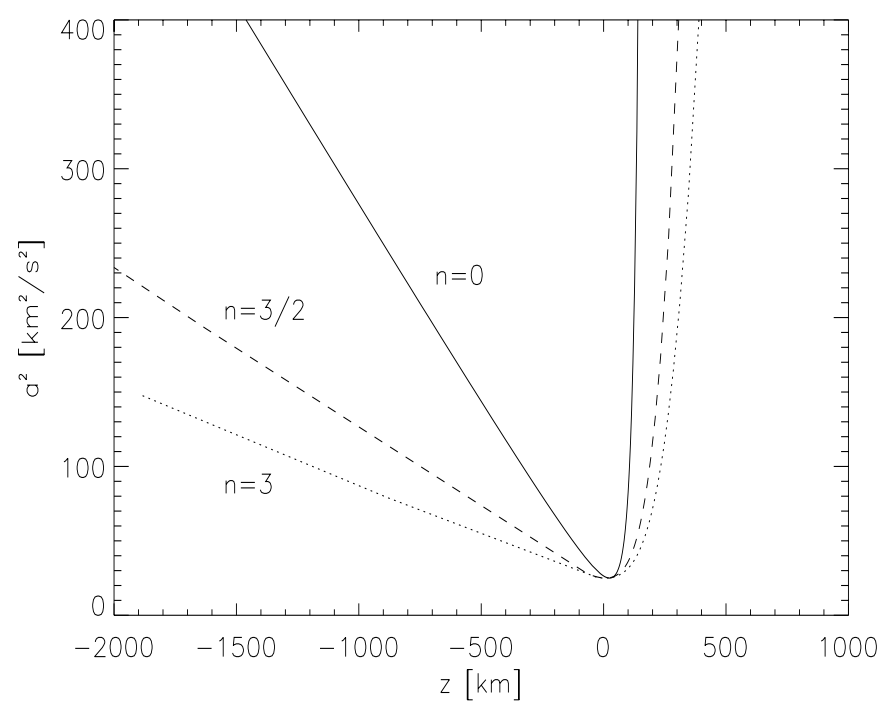

Fig. 2. Squares of isothermal sound speeds as functions of the geometrical height $z$. Displayed by solid, dashed-dotted, and dotted curves.

Here, $\alpha$ is related to the polytropic index $n$ by

$\alpha=2-\frac{1}{1+n}$

For $0 \leq n<\infty$ we have $1 \leq \alpha<2$.

Figure 2 shows stratifications $a^{2}(z)$ for $n=0, n=3 / 2$, and $n=3$. Here, the mass of the minimum of the sound speeds $a$ and the minimum value $a_{\mathrm{min}}$ are fixed. The values of $m_{0}$ and $\epsilon$ are calculated. We have $m_{0}=1 / 2(2-\alpha) m_{\min }$.

Layers with $n=3 / 2$ or $n=3$ are more realistic than a layer with $n=0$. However, to present a wave equation with exact solutions, we have no option but $n=0$.

\section{The reduction of the adiabatic wave equation}

Let $\Delta p(z, t)$ be the Lagrangian pressure perturbation, $c$ the adiabatic sound speed. The frequency is denoted by $\omega$, the horizontal wave number by $k$. We study adiabatic waves with time dependence $\exp ( \pm \mathrm{i} \omega t)$. The time-independent wave equation of the Lagrangian pressure perturbation $\Delta p$ is

$$
\begin{aligned}
& \omega^{2} c^{2}\left[\frac{\mathrm{d}^{2} \Delta p}{\mathrm{~d} z^{2}}-\frac{1}{\rho} \frac{\mathrm{d} \rho}{\mathrm{d} z} \frac{\mathrm{d} \Delta p}{\mathrm{~d} z}\right] \\
& -\left[k^{2} g\left[g+\frac{c^{2}}{\rho} \frac{\mathrm{d} \rho}{\mathrm{d} z}\right]+\omega^{2}\left(c^{2} k^{2}-\omega^{2}\right)\right] \Delta p=0 .
\end{aligned}
$$

(Schmitz \& Fleck 1994, Eq. (5). The reason for preferring the Lagrangian pressure perturbation is explained there.) Instead of the geometrical height $z$ we use the column mass $m$ as the independent variable. For the classical ideal gas with $p=a^{2} \rho$ and $c^{2}=\gamma a^{2}$, where $\gamma$ is the adiabatic exponent, the wave equation takes the form (cf. Schmitz \& Steffens 1999)

$$
\begin{aligned}
& m^{2} \frac{\mathrm{d}^{2} \Delta p}{\mathrm{~d} m^{2}}+\frac{\Delta p}{\gamma \omega^{2} g^{2}} \\
& \cdot\left[\left[\omega^{4}-k^{2} g^{2}(1-\gamma)\right] a^{2}-\omega^{2} \gamma a^{4} k^{2}-k^{2} g^{2} \gamma m \frac{\mathrm{d} a^{2}}{\mathrm{~d} m}\right]=0 .
\end{aligned}
$$

For $a^{2}(m)$ given by Eq. (1) we obtain

$\frac{\mathrm{d}^{2} \Delta p}{\mathrm{~d} m^{2}}$

$+\left(\frac{A}{m-m_{0}}+\frac{B}{\left(m-m_{0}\right)^{2}}+\frac{C m}{\left(m-m_{0}\right)^{2}}+\frac{D m^{2}}{\left(m-m_{0}\right)^{2}}\right) \Delta p=0$,

with the coefficients

$A=\epsilon \frac{\omega^{4}-k^{2} g^{2}(1-\gamma)}{\gamma \omega^{2} g^{2}}$,

$B=\frac{2 \epsilon m_{0} k^{2}}{\omega^{2}}, \quad C=-\epsilon \frac{k^{2}}{\omega^{2}}, \quad D=-\epsilon^{2} \frac{k^{2}}{g^{2}}$.

This differential equation can be reduced to Whittaker's equation. Introducing a new independent variable $\xi$ by

$\xi=m-m_{0}$,

some decompositions lead to the equation

$\frac{\mathrm{d}^{2} \Delta p}{\mathrm{~d} \xi^{2}}$

$+\left(-\epsilon^{2} \frac{k^{2}}{g^{2}}+\frac{A+C+2 m_{0} D}{\xi}+\frac{B+C m_{0}+D m_{0}^{2}}{\xi^{2}}\right) \Delta p=0$.

Now we introduce the independent variable $x$ by

$x=\frac{2 \epsilon k}{g} \xi$

where now $k \neq 0$. Finally, we obtain the equation

$\frac{\mathrm{d}^{2} \Delta p}{\mathrm{~d} x^{2}}$

$+\left(-\frac{1}{4}+\frac{g}{2 \epsilon k} \frac{A+C+2 m_{0} D}{x}+\frac{B+C m_{0}+D m_{0}^{2}}{x^{2}}\right) \Delta p=0$.

This is Whittaker's differential equation

$\frac{\mathrm{d}^{2} \Delta p}{\mathrm{~d} x^{2}}+\left(-\frac{1}{4}+\frac{\kappa}{x}+\frac{\frac{1}{4}-\mu^{2}}{x^{2}}\right) \Delta p=0$

where the coefficients $\kappa$ and $\mu$ are given by

$\kappa=\frac{\omega^{4}-2 \epsilon m_{0} \gamma k^{2} \omega^{2}-k^{2} g^{2}}{2 k g \gamma \omega^{2}}$

$\mu= \pm \frac{1}{2 \omega g} \sqrt{\left(g^{2}+4 \epsilon^{2} m_{0}^{2} k^{2}\right) \omega^{2}-4 \epsilon m_{0} k^{2} g^{2}}$.

The parameters $\epsilon$ and $m_{0}$ occur in the coefficients $\kappa$ and $\mu$ as a product $\epsilon m_{0}$. For real $\omega^{2}$, the coefficient $\kappa$ is real, $\mu$ is real or imaginary. For $\mu=0$ we obtain a relation $\omega(k)$ The corresponding curve is a boundary line of the $k-\omega$-diagram. 


\section{The solutions of the wave equation}

Two independent solutions of Whittaker's equation are (Abramowitz \& Stegun 1965)

$\Delta p_{1}(x)=p_{* 1} \mathrm{e}^{-x / 2} x^{+\mu+\frac{1}{2}} M\left(\frac{1}{2}+\mu-\kappa, 1+2 \mu, x\right)$,

$\Delta p_{2}(x)=p_{* 2} \mathrm{e}^{-x / 2} x^{-\mu+\frac{1}{2}} M\left(\frac{1}{2}-\mu-\kappa, 1-2 \mu, x\right)$,

where now

$x=\frac{2 \epsilon k}{g}\left(m-m_{0}\right)$.

$M(\alpha, \beta, x)$ is a confluent hypergeometric function, $p_{* 1}$ and $p_{* 2}$ are arbitray constant pressures. When $\mu$ is imaginary, $\Delta p_{1}$ and $\Delta p_{2}$ are complex conjugate. In this case, together with the factors $\exp ( \pm \mathrm{i} \omega t)$ they represent travelling waves. When $\mu$ is real, we may assume that $\mu>0$. The solution must fulfill certain boundary conditions. We demand that the pressure perturbation $\Delta p$ vanishes at infinity:

$\Delta p \rightarrow 0 \quad$ as $z \rightarrow \infty$,

which is equivalent to

$\Delta p \rightarrow 0$ for $m \rightarrow m_{0}$ or $x \rightarrow 0$.

For $z \rightarrow-\infty$ we require vanishing pressure perturbations. This assumption is common. It corresponds to the condition of vanishing non-radial pressure perturbations in the center of a star. Therefore,

$\Delta p \rightarrow 0 \quad$ as $\quad z \rightarrow-\infty$,

which is equivalent to

$\Delta p \rightarrow 0 \quad$ as $m \rightarrow \infty$ or $\quad x \rightarrow \infty$.

We have $M(\alpha, \beta, x)=1$ at $x=0$. Thus, the first solution $\Delta p_{1}$ is appropriate. We get

$\Delta p_{1}(x)=p_{* 1} x^{\mu+\frac{1}{2}} \rightarrow 0$ for $x \rightarrow 0$.

The second solution behaves as

$\Delta p_{2}(x)=p_{* 2} x^{-\mu+1 / 2}$ for $x \rightarrow 0$.

It diverges when $\mu>1 / 2$. One can show that the case $\mu<1 / 2$ does not occur in the $k-\omega$ region of the p-modes. Therefore, we have to take the first solution. In this case the pressure perturbation behaves as

$\Delta p_{1}(z)=p_{*} \exp \left[-\frac{g}{\epsilon m_{0}}\left(\frac{1}{2}+\mu\right) z\right] \quad$ as $\quad z \rightarrow \infty$.

When $z \rightarrow-\infty$ or $x \rightarrow \infty$ the asymptotic behavior of $M$ is (Abramowitz \& Stegun 1965)

$M(\alpha, \beta, x)=\frac{\Gamma(\beta)}{\Gamma(\alpha)} \mathrm{e}^{x} x^{\alpha-\beta} \quad$ as $\quad x \rightarrow \infty$.

We obtain

$\Delta p_{1}(x)=p_{* 1} \frac{\Gamma(1+2 \mu)}{\Gamma\left(\frac{1}{2}+\mu-\kappa\right)} x^{-\kappa} \mathrm{e}^{x / 2} \quad$ as $\quad x \rightarrow \infty$.

As $\Gamma(y) \rightarrow \infty$ for $y \rightarrow 0,-1,-2, \ldots$, the condition of convergence of $\Delta p_{1}(x)$ at $x \rightarrow \infty$ is:

$\frac{1}{2}+\mu-\kappa=-j, \quad j=0,1,2,3 \ldots$

In this case, the function $M$ reduces to a polynomial of degree $j$, a generalized Laguerre-polynomial, and the pressure perturbation $\Delta p_{1}$ decays exponentially for $x \rightarrow \infty$.

\section{The dispersion relation of the modes}

Taking the square of

$\mu=\kappa-j-\frac{1}{2}$

where $j=0,1,2, \ldots$ is the vertical wave number, inserting the coefficients $\kappa$ and $\mu$ as given by Eqs. (20) and (21), and putting

$y=\frac{\omega^{2}}{k g} \quad$ and $\quad k_{0}=\frac{g}{4 \gamma \epsilon m_{0}}$,

we finally obtain the dispersion relation, a fourth order equation for $\omega^{2}$ :

$y^{4}-\left\{2 \gamma(1+2 j)+\frac{k}{k_{0}}\right\} y^{3}$

$-\left\{2\left(1-2 \gamma^{2} j(j+1)\right)-\gamma(1+2 j) \frac{k}{k_{0}}\right\} y^{2}$

$+\left\{2 \gamma(1+2 j)+(1+\gamma) \frac{k}{k_{0}}\right\} y+1=0$.

For $j=0$, a solution is $y=-1$. Separating this solution, for $j=0$, the dispersion relation can immediately be reduced to an equation of third order:

$y^{3}-\left\{(2 \gamma+1)+\frac{k}{k_{0}}\right\} y^{2}$

$+\left\{(2 \gamma-1)+(\gamma+1) \frac{k}{k_{0}}\right\} y+1=0$.

For $m \rightarrow \infty$ or $z \rightarrow-\infty$ the structure of our equilibrium configuration approaches the structure of a polytropic layer with index $n=0$. For a polytropic convection zone without an overlying atmosphere we have

$a^{2}(z)=-\frac{g z}{1+n} \quad$ with $\quad-\infty<z \leq 0$.

The modes of the polytropic layer were investigated by Lamb (1932). In this case, by the boundary conditions

$\Delta p=0 \quad$ at $\quad z \rightarrow-\infty$ and $z=0$

one obtains a dispersion relation from which in the case $n=0$

$\omega^{2}=k g\left(\gamma(j+1)+\sqrt{[\gamma(j+1)]^{2}+1}\right)$

for the frequencies of the stable p-modes. This relation can also be obtained from Eq. (37) for $k_{0} \rightarrow \infty$.

\section{Discussion of the dispersion relations}

The dispersion relation (37) is an equation of the fourth degree in $\omega^{2}$. Therefore, we need not go into details of the calculation. For each vertical wave number $j$ we obtain two branches $\omega^{2}(k)$. There are stable p-modes and unstable convective modes. We now put $\gamma=5 / 3$. The gravity $g$ and the parameters $\epsilon$ and $m_{0}$ are given in Sect. 2. Figure 3 shows the ridges of the p-modes for $j=0,1,2,3$ and the $\mathrm{p}$-modes of the corresponding pure convection zone. The qualitative behavior of the p-modes is independent of the parameters $\epsilon$ and $m_{0}$. We find that the curvature of the $\mathrm{p}_{i}$-ridges with $i=1,2,3, \ldots$ is monotonous. Only the ridge 


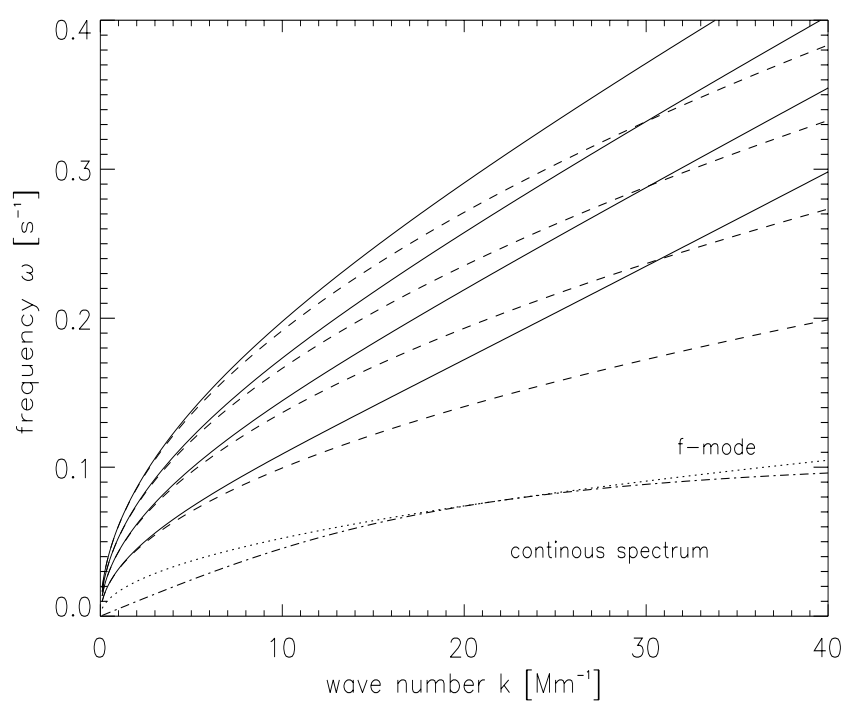

Fig. 3. p-modes of the model (solid lines) and of the pure convection zone (dashed lines). Further, the f-mode (dotted line) and the limiting curve of the continuum (dashed-dotted).

of the $\mathrm{p}_{0}$-modes has an inflection point at $k \approx 18.6 \mathrm{Mm}^{-1}$. Here, this ridge bends. This effect is poorely distinct, but interesting for the following reason. Steffens \& Schmitz (2000) have investigated the behavior of the p-modes of a series of modified VAL$\mathrm{C}$-models of the solar atmosphere. They find bending of the ridges when a (hot or cool) chromosphere is present. The case of an isothermal atmosphere with a height of $600 \mathrm{~km}$ (Fig. $3 \mathrm{~b}$ of Steffens \& Schmitz) shows the beginning of the bending of the $\mathrm{p}_{0}$-mode.

For higher wave numbers $k$, the dispersion curves become linear. In the limit, we have:

$$
\omega=c_{*} k \quad \text { as } \quad k \rightarrow \infty,
$$

with a fixed adiabatic sound speed $c_{*}$. For $k \rightarrow \infty$, the dispersion relation (37) reduces to

$y^{4}-\frac{k}{k_{0}} y^{3}+\gamma(2 j+1) \frac{k}{k_{0}} y^{2}+(\gamma+1) \frac{k}{k_{0}} y+1=0$.

Inserting $\omega=c_{*} k$, we see that the first two terms are the leading ones when $k \rightarrow \infty$. We obtain

$c_{*}=c_{\min }=\sqrt{4 \epsilon \gamma m_{0}}$,

where $c_{\min }$ is the minimum of the adiabatic sound speed at the position $m=2 m_{0}$. So for high wavenumbers the modes $\Delta p(z)$ should concentrate to the position of the minimum. For $k \rightarrow \infty$, the Exponent $\mu$ as given by Eq. (21) is

$\mu=\frac{\epsilon m_{0}}{g} k$

As can be shown readily, the amplitude of the pressure perturbation $\Delta p_{1}$ becomes peak-like at $2 m_{0}$ :

$\mathrm{e}^{-x / 2} x^{\mu+1 / 2} \propto \exp (-2 \epsilon m k / g)\left(m-m_{0}\right)^{2 \epsilon m_{0} k / g}$

as $k \rightarrow \infty$. This function is maximum at $m=2 m_{0}$. In the limit, the modes concentrate at the position of the minimum.

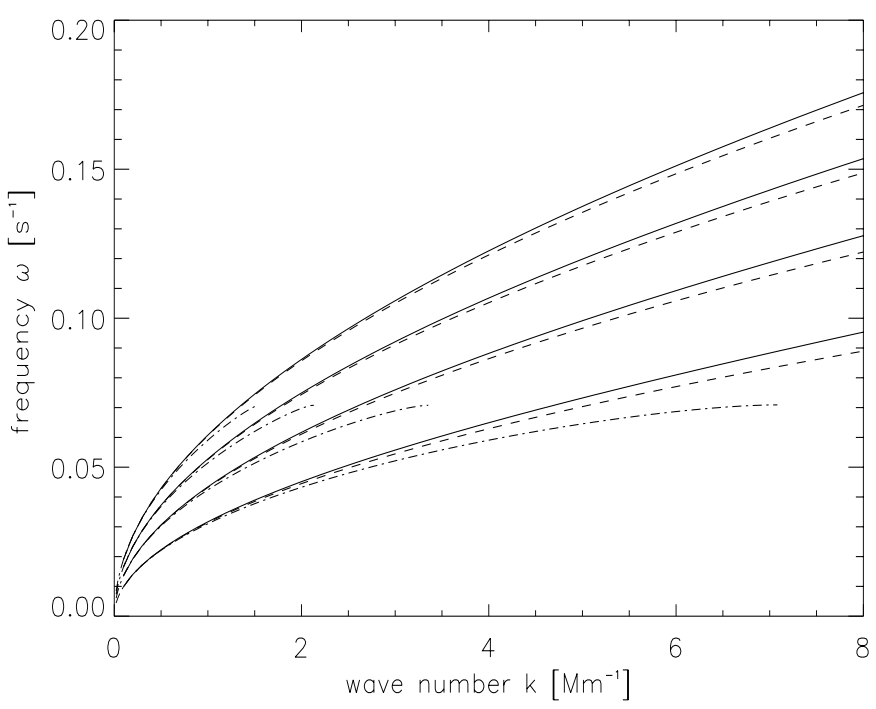

Fig. 4. p-modes of the present model (solid), of the pure convection zone (dashed), and of a convection zone with an isothermal atmosphere (dashed-dotted).

\section{Relations to the modes of a polytropic layer with an overlying isothermal atmosphere}

Schmitz \& Steffens (1999) have studied the behavior of waves in a plane layer with

$a^{2}(m)=\epsilon m^{\lambda}+a_{0}^{2}$

This stratification represents a polytropic layer smoothly joined by an asymptotically isothermal atmosphere. The exponent $\lambda$ is given by $\lambda=1 /(1+n)$. As in the case of the present paper, the adiabatic wave equation can be reduced to Whittaker's equation, and the dispersion relation of the modes is a fourth order algebraic equation in $\omega^{2} / g k$. For $n=0$ we have

$a^{2}(m)=\epsilon m+a_{0}^{2}$.

For $m \rightarrow \infty$ both the stratifications (1) and (48) approach the limit $a^{2}=\epsilon m$. In the case of the stratification (48) the independent variable is $\xi=m$ instead of $\xi=m-m_{0}$. The coefficient $\kappa$ belonging to the stratification (48) reads (Schmitz \& Steffens 1999, Eq. (22))

$\kappa=\frac{\omega^{4}-2 \gamma a_{0}^{2} \omega^{2} k^{2}-k^{2} g^{2}}{2 k g \gamma \omega^{2}}$.

The coefficients (20) and (49) are equal when $\epsilon m_{0}=a_{0}^{2}$. Figure 1 shows the corresponding function $a^{2}(z)$. By varying the sound speed $a_{0}$ one can show that the case $\epsilon m_{0}=a_{0}^{2}$ gives the best approximation of the stratification (48) to the stratification (1) below the minimum. For example, Fig. 1 also displays the case $a_{0}^{2}=4 \epsilon m_{0}$, where the sound speed of the isothermal atmosphere is equal to the minimum sound speed of the stratification (1). Figure 4 shows dispersion curves of the pure convection zone, the present model and of the stratification (48) with $a_{0}^{2}=\epsilon m_{0}$. All ridges of the layer with the isothermal atmosphere lie below the ridges of the pure convection zone. This behavior is independent of the numerical value of $a_{0}$. The p-mode ridges of the present model, however, are above the corresponding ridges of the pure convection zone. 


\section{The continuous spectrum}

For real frequencies $\omega$, the coefficient $\mu$ defined by Eq. (21) is real or imaginary. For $\mu=0$ we obtain a relation $\omega=\omega_{0}(k)$ with

$\omega_{0}(k)=2 k g \sqrt{\frac{\epsilon m_{0}}{g^{2}+4 \epsilon^{2} m_{0}^{2} k^{2}}}$.

For $\omega<\omega_{0}$, the coefficient $\mu$ is imaginary, so that the spectrum becomes continous. The solutions (22) and (23) represent the amplitudes of propagating waves. Besides the p-modes, Fig. 3 shows the f-mode $\omega^{2}=k g$ und the limiting curve $\omega_{0}(k)$. The f-mode touches the curve $\omega_{0}(k)$ at $k=g / 2 \epsilon m_{0}$.

The function $\omega_{0}(k)$ can be obtained from the asymptotic form of the wave equation. By use of the height $z$, with $\rho=$ $\gamma p / c^{2}$, the wave equation (10) reads

$\omega^{2} c^{2} \frac{\mathrm{d}^{2} \Delta p}{\mathrm{~d} z^{2}}+\omega^{2}\left(\gamma g+\frac{\mathrm{d} c^{2}}{\mathrm{~d} z}\right) \frac{\mathrm{d} \Delta p}{\mathrm{~d} z}$

$+\left\{k^{2} g\left(g(\gamma-1)+\frac{\mathrm{d} c^{2}}{\mathrm{~d} z}\right)-\omega^{2}\left(c^{2} k^{2}-\omega^{2}\right)\right\} \Delta p=0$.

For $z \rightarrow \infty, a^{2}$ is given by Eq. (5), so that $c^{2}(z) \rightarrow c_{0}^{2} \mathrm{e}^{z / h}$ with $h=\epsilon m_{0} / g$ and $c_{0}^{2}=\gamma \epsilon m_{0}$. For fixed $\omega$ and $z \rightarrow \infty$ we obtain the equation

$\omega^{2} \frac{\mathrm{d}^{2} \Delta p}{\mathrm{~d} z^{2}}+\frac{\omega^{2}}{h} \frac{\mathrm{d} \Delta p}{\mathrm{~d} z}+\left(\frac{g k^{2}}{h}-k^{2} \omega^{2}\right) \Delta p=0$,

the solutions of which reads

$\Delta p(z)=\exp \left(-\frac{z}{2 h}\right) \exp ( \pm \mathrm{i} K z)$,

where

$K=\sqrt{\frac{g}{h} \frac{k^{2}}{\omega^{2}}-k^{2}-\frac{1}{4 h^{2}}}$.

For $K=0$ we obtain the relation (50). The pressure perturbation (53) is independent of $\gamma$. That means the waves which are acoustic in the convection zone turn into gravity waves in the range of the exponential temperature increase.

In conclusion, let us discuss whether there are resonances in the continous region. Here, the solution $U$ of Whittaker's equation is appropriate:

$\Delta p(x)=p_{*} \mathrm{e}^{-x / 2} x^{\mu+\frac{1}{2}} U\left(\frac{1}{2}+\mu-\kappa, 1+2 \mu, x\right)$.

When $z \rightarrow-\infty$ or $x \rightarrow \infty$, the asymptotic behavior of $U$ is (Abramowitz \& Stegun 1965)

$U(a, b, x)=x^{-a} \quad$ as $\quad x \rightarrow \infty$,

so that

$\Delta p \rightarrow p_{*} \mathrm{e}^{-x / 2} x^{K} \quad$ as $\quad x \rightarrow \infty$.

For real $\omega$, the coefficient $\kappa$ is real. The pressure perturbation $\Delta p$ decays for $x \rightarrow \infty$ or $m \rightarrow \infty$. As a function of $\omega, \Delta p$ is smooth and non-oscillatory for $x \rightarrow \infty$.

Let us now discuss the behavior of the solution for $z \rightarrow \infty$. For $x \rightarrow 0$ where

$U(x)=\frac{\Gamma(-2 \mu)}{\Gamma\left(\frac{1}{2}-\kappa-\mu\right)}+\frac{\Gamma(+2 \mu)}{\Gamma\left(\frac{1}{2}-\kappa+\mu\right)} x^{-2 \mu}$
(Abramowitz \& Stegun 1965) we have:

$\Delta p=p_{*} x^{1 / 2}\left[\frac{\Gamma(-2 \mathrm{i} \alpha)}{\Gamma\left(\frac{1}{2}-\kappa-\mathrm{i} \alpha\right)} x^{\mathrm{i} \alpha}+\frac{\Gamma(+2 \mathrm{i} \alpha)}{\Gamma\left(\frac{1}{2}-\kappa+\mathrm{i} \alpha\right)} x^{-\mathrm{i} \alpha}\right]$.

with $\mu=\mathrm{i} \alpha$. The amplitude of the pressure perturbation is

$|\Delta| p \propto x^{1 / 2}\left|\frac{\Gamma(2 \mathrm{i} \alpha)}{\Gamma\left(\frac{1}{2}-\kappa+\mathrm{i} \alpha\right)}\right|$.

The identity $|\Gamma(\mathrm{i} y)|^{2}=\pi / y \sinh (\pi y)$ shows that $|\Gamma(2 \mathrm{i} \alpha)|$ is a smooth function of $\alpha$, and thus of $\omega$. We finally are left with the part

$|\Delta p| \propto \frac{1}{\left|\Gamma\left(\frac{1}{2}-\kappa+\mathrm{i} \alpha\right)\right|}$.

Because of the poles of the $\Gamma$-function at $0,-1,-2, \ldots$, the function $\left|\Gamma\left(\frac{1}{2}-\kappa+\mathrm{i} \alpha\right)\right|$ varies essentially only for $\kappa>1 / 2$ and $|\alpha| \ll 1$. If strong variations are present, then for $|\alpha| \ll 1$, that means, at and below the curve $\omega_{0}(k)$. However, $\kappa$ is negative at and directely below this curve, as can be shown readily. Therefore, there are no significant variations and thus no resonances.

\section{Vertically propagating waves}

As this case is not very interesting, we shall be brief. For $k=0$, the wave Eq. (11) reduces to the equation

$m^{2} \frac{\mathrm{d}^{2} \Delta p}{\mathrm{~d} m^{2}}+\frac{\omega^{2} a^{2}(m)}{\gamma g^{2}} \Delta p=0$.

For the more general function $a^{2}(m)$ given by Eq. (7) we obtain:

$\frac{\mathrm{d}^{2} \Delta p}{\mathrm{~d} m^{2}}+\frac{\omega^{2} \epsilon}{\gamma g^{2}}\left(m-m_{0}\right)^{-\alpha} \Delta p=0$.

The solution fulfilling the condition $\Delta p=0$ at $m=m_{0}$ is a standing wave

$\Delta p=p_{*} \sqrt{m-m_{0}} J_{1 / v}\left(2 \frac{\omega}{g} \sqrt{\frac{\epsilon}{\gamma}} \frac{1}{v}\left(m-m_{0}\right)^{v / 2}\right)$

where $J_{1 / v}$ is a Bessel function of the first kind, and $v=2-\alpha$ so that $0 \leq v<1$ if $\alpha \leq 1<2$. We have $\Delta p \propto m-m_{0}$ for $m \rightarrow m_{0}$. In terms of the mass $m, \Delta p$ behaves as the pressure perturbation of the pure convection zone with $m_{0}=0$. For $a^{2}(m)$ given by Eq. (1) we obtain $v=1$.

\section{Conclusions}

We have dealt with the behavior of p-modes of a plane layer with constant gravity. The layer consists of a polytropic convection zone with polytropic index $n=0$ smoothly joined by a hot envelope. The fact that the convection zone is convectively unstable is not essential as we study only p-modes. Besides, the behavior of the p-modes of a pure convection zone is qualitatively independent of the polytropic index. We have $\omega^{2} \propto g k$. All results, the form of the modes and the dispersion relation are given in closed form. We discussed the deviations of the ridges from the ridges of a pure convection zone. Also a comparison of the ridges with those of a convection zone with a smoothly joined isothermal atmosphere was performed. In the $k-\omega$ diagram, there is a continuum below the f-mode $\omega^{2}=g k$. The corresponding waves are propagating gravity waves. A study of the 
amplitudes of these waves by asymtotic representations showed that there are no resonances in the continuum. The case of purely vertical wave propagation is very simple. The solution was presented for a more general temperature stratification, namely for a convection zone with arbitrary positive index. We did not investigate convectively unstable modes. A study of such modes could give information about the influence of a hot envelope on the behavior of the convection.

\section{References}

Abramowitz M., \& Stegun I. A. 1965, Handbook of Mathematical functions (New York: Dover Publ. Inc.)
Antia H. M., \& Basi S. 1999, ApJ, 519, 400

Carlson M., \& Stein R. F. 1995, APJ, 440, L29

Chiuderi C., \& Giovanardi C. 1979, Sol. Phys., 64, 27

Fleck B., \& Schmitz F. 1993, A\&A, 273, 671

Lamb H. 1932, Hydrodynamics (Cambridge: University Press)

Pekeris C. L. 1938, ApJ, 88, 189

Pekeris C. L. 1948, Phys. Rev., 73, 145

Price G. 1996, ApJ, 458, 850

Schmitz F., \& Fleck B. 1994, A\&AS, 106, 129

Schmitz F., \& Steffens S. 1999, A\&A, 344, 973

Schmitz F., \& Steffens S. 2000, A\&A, 356, 319

Steffens S. 1998, Thesis, University of Würzburg

Steffens S., \& Schmitz F. 2000, A\&A, 354, 280

Vernazza J., Avrett E., \& Loeser R. 1981, ApJS, 45, 635 